\title{
Generalidad de la Hipótesis de la Flexibilidad de Procesamiento en el Aprendizaje Humano: Del Condicionamiento al Aprendizaje Predictivo
}

\section{Generality of the Flexibility of Processing Hypothesis in Human Learning: From Conditioning to Predictive Learning}

\author{
Gonzalo R. Quintana, Fernando P. Ponce y Edgar H. Vogel \\ Universidad de Talca
}

\begin{abstract}
En un experimento de aprendizaje predictivo humano se investigó si un estímulo visual compuesto por 2 elementos es procesado como un todo (configuracionismo) o como la suma de sus elementos (elementalismo). El experimento se realizó a través de un juego computacional en el que los participantes debían aprender que ciertos microorganismos (claves) compuestos por 2 rasgos visuales (elementos) producían alergia en animales ficticios. Un total de 38 estudiantes universitarios aprendieron que 2 microorganismos causaban alergia (claves positivas) y otros 2 no (claves negativas). Posteriormente, se examinó el valor predictivo que los participantes asignaban a un nuevo microorganismo compuesto por un elemento de cada clave positiva y a otro compuesto por un elemento de cada clave negativa. El valor predictivo asignado a las nuevas claves fue similar al asignado a sus respectivas claves aprendidas cuando los elementos eran perceptivamente separables (tamaño y ángulo de la figura), indicando elementalismo, pero no cuando eran integrales (brillo y saturación), indicando configuracionismo. Esto apoya la hipótesis de que las características de los estímulos determinan el tipo de procesamiento en el aprendizaje.
\end{abstract}

Palabras clave: configuracionismo, elementalismo, aprendizaje, integralidad, separabilidad

An experiment on human predictive learning investigated whether a visual stimulus composed of 2 elements is processed as a whole (configural processing) or as the aggregation of its elements (elemental processing). The experiment was conducted by means of a computer game in which the participants have to learn that certain microorganisms (cues) composed of 2 visual features (elements) produced an allergic reaction in fictitious animals. A total of 38 college students learned that 2 microorganisms cause allergy (positive cues) and 2 microorganisms do not (negative cues). Subsequently, the predictive value that participants assigned to a new microorganism composed of one element of each positive cue and to a new microorganism composed of one element of each negative cue was examined. The results indicated that the predictive ratings of the new cues were similar to those of their respective learned cues when the dimensions were perceptually separable (size and angle), indicating elemental processing, but not when they were integral (brightness and saturation), indicating configural processing. These results support the hypothesis that stimulus characteristics determine the type of processing in learning.

Keywords: configurationism, elementalism, learning, integrality, separability

Varias revisiones recientes en el ámbito del aprendizaje asociativo coinciden en señalar que uno de los problemas teórico-empíricos más fundamentales en la actualidad se refiere a cómo debe concebirse la representación efectiva de los estímulos que participan en la asociación (e.g., Harris, 2006, 2010; Melchers, Shanks \& Lachnit, 2008; Vogel \& Ponce, en prensa; Wagner \& Vogel, 2009). La mayoría de la investigación que ha abordado esta problemática se ha realizado con los denominados estímulos compuestos, los Chile.

Gonzalo R. Quintana, Fernando P. Ponce y Edgar H. Vogel, Facultad de Psicología, Universidad de Talca,

El presente estudio fue financiado a Edgar Vogel por el proyecto FONDECYT № 1060838 y por la Universidad de Talca, a través del programa de investigación "Calidad de Vida y Ambientes Saludables" (Res. 387/2007).

La correspondencia relativa a este artículo debe ser dirigida a Edgar H. Vogel, Facultad de Psicología, Universidad de Talca, Avda. Lircay s/n, Talca, Chile. E-mail: evogel@utalca.cl 
cuales consisten en la presentación conjunta de dos o más estímulos perceptivamente distinguibles, tales como un sonido y una imagen. El interés de los investigadores se ha focalizado en determinar cuál es la relación que existe entre las representaciones de los estímulos por sí solos en comparación con la representación del compuesto.

La visión más simple plantea que la representación de un conglomerado de estímulos que se presentan juntos es equivalente a la suma de las representaciones de cada estímulo por separado. El rasgo distintivo de este enfoque es su carácter aditivo, en el que se supone que cada estímulo mantiene la totalidad de su representación al formar un compuesto con otro estímulo. Por ejemplo, si un animal es entrenado en condicionamiento clásico con un estímulo condicionado A seguido por un estímulo incondicionado (EI) y un estímulo condicionado B también seguido por el EI, se supone que, al presentar ambos estímulos juntos (AB), la repuesta condicionada debería ser equivalente a la suma de las respuestas a los estímulos A y B por sí solos. Existe evidencia experimental congruente con esta noción de sumatoria de fuerzas asociativas (Whitlow \& Wagner, 1972).

Naturalmente, esta simple aditividad de los estímulos que forman compuestos es solamente una idealización de la relación que existe entre la representación de un compuesto y sus componentes, como claramente lo demuestran ciertos experimentos de discriminación, en los cuales los animales aprenden asociaciones que son específicas de determinadas conjunciones de estímulos. Por ejemplo, se ha demostrado que diversos animales pueden aprender la discriminación patrón negativo (Harris, Livesey, Gharaei \& Westbrook, 2008; Ludwig \& Lachnit, 2003; Whitlow \& Wagner, 1972), en la cual dos estímulos condicionados A y B son seguidos por un EI cuando se presentan por sí solos (denotados como $\mathrm{A}+\mathrm{y} \mathrm{B}+$ ), pero no cuando se presentan en conjunto (denotado como AB-). Ya que A y B desarrollan una asociación con el EI, la representación de $\mathrm{AB}$ debería ser, en algún grado, distinta a la mera suma de sus elementos para que la discriminación se resuelva. Otro ejemplo es la discriminación bicondicional, en la cual dos compuestos, $\mathrm{AB}$ y $\mathrm{CD}$, son reforzados (es decir, seguidos por el EI) mientras que dos compuestos AC y BD no lo son (Harris et al., 2008; Harris \& Livesey, 2008; Saavedra, 1975). Ya que los componentes A, B, C y D son reforzados parcialmente, en este caso la discriminación bicondicional no puede ser resuelta en base a la asociación de sus elementos, concluyéndose que la representación de un compuesto es distinta a la suma de sus elementos constituyentes.

En respuesta a estos fenómenos, se ha concluido que la relación entre compuestos y sus componentes no es siempre lineal, como podría indicarlo el fenómeno de sumatoria. Por ejemplo, Wagner y Rescorla (1972) propusieron que cada vez que dos estímulos se presentan juntos, además de activarse las unidades representacionales de cada estímulo, se activa una unidad especial única de esa configuración de estímulos, la cual lo hace cada vez que los estímulos ocurren en compuesto. Otros modelos más recientes han sugerido formas más radicales en las que difieren las representaciones de los estímulos al formar parte de diferentes compuestos (Harris, 2006, 2010; Harris \& Livesey, 2010; McLaren \& Mackintosh, 2002; Pearce, 2002; Wagner, 2003). Aunque estos modelos difieren importantemente en la forma en cómo conciben la conjunción de estímulos, todos coinciden en un principio general, que usualmente se denomina sensibilidad contextual, el cual denota el hecho de que la representación de cualquier estímulo definido externamente por el experimentador cambia al ser presentado en distintos contextos de estimulación. Así, por ejemplo, la representación de $\mathrm{A}$ en un contexto que contiene a $\mathrm{B}$ no es la misma que la representación de $\mathrm{A}$ en un contexto que no lo contiene. También se suelen utilizar los términos procesamiento configuracional para referirse a la sensibilidad contextual y elementalista para referirse a su ausencia (Wagner, 2003).

Un aspecto interesante de estas representaciones contextualmente sensitivas es que solo basta con un mínimo de sensibilidad contextual para que las discriminaciones del tipo patrón negativo y bicondicional se aprendan exitosamente. Otros hallazgos, sin embargo, sugieren que la sensibilidad 
contextual debería ser mucho mayor en ciertas circunstancias (e.g., Díaz, Torres, Castillo, Cornejo \& Vogel, 2009; Pearce \& Wilson, 1991). Un ejemplo ilustrativo de esta cuestión es el procedimiento de sumatoria, donde dos estímulos se refuerzan por separado $(\mathrm{A}+\mathrm{y} \mathrm{B}+)$ y luego se examina el nivel de respuesta al compuesto $\mathrm{AB}$. Si se supone una representación altamente sensible al contexto, donde $\mathrm{AB}$ tiene muy poco en común con $\mathrm{A}$ y $\mathrm{B}$, se esperaría que el nivel de respuesta a $A B$ sea igual o inferior a $A$ y B. Por el contrario, si se supone una representación con poca sensibilidad contextual, se espera que mucho de lo aprendido acerca de $A$ y $B$ se transfiera a $A B$, obteniendo una respuesta mayor al compuesto que a sus elementos sumados. Los hallazgos con este procedimiento han sido controversiales, obteniéndose tanto sumatoria (Collins \& Shanks, 2006; Cornejo, Castillo, Saavedra \& Vogel, 2010; Díaz et al., 2009; Rescorla, 1997; Soto, Vogel, Castillo \& Wagner, 2009; Whitlow \& Wagner, 1972) como su ausencia (Aydin \& Pearce, 1995; Rescorla \& Coldwell, 1995).

Además de los resultados contrastantes obtenidos con el procedimiento de sumatoria, otras tareas de condicionamiento arrojan el mismo nivel de ambigüedad (e.g., Díaz et al., 2009). Frente a esto, algunos investigadores han propuesto una hipótesis de flexibilidad de procesamiento, la cual señala que la sensibilidad contextual o el tipo de procesamiento de estímulos varía de acuerdo a ciertas circunstancias, tales como la naturaleza de los estímulos, el tipo de tarea, las características individuales de los participantes y la experiencia previa (e.g., Melchers et al., 2008).

Dentro de esta miríada de alternativas, el tipo de estímulos es quizá uno de los factores de flexibilidad de procesamiento que aparece como más natural en situaciones de condicionamiento. Pese a esto, muy pocas investigaciones han examinado sistemáticamente esta posibilidad. En uno de estos estudios, realizado con el procedimiento de condicionamiento de la membrana nictitante en conejos, Kehoe, Horne, Horne y Macrae (1994) entrenaron separadamente a los animales con un tono, un ruido y una luz seguidos por el EI y luego examinaron el nivel de respuesta a las tres combinaciones de dos estímulos, en comparación con los estímulos aislados. Los resultados arrojaron sumatoria, es decir, mayor respuesta al compuesto que a los elementos, cuando el compuesto estaba formado por estímulos de distinta modalidad sensorial (e.g., tono-luz y ruido-luz), pero no se observó dicha sumatoria con el compuesto intradimensional (tono-ruido).

Los hallazgos de Kehoe et al. (1994) y otros estudios similares (Honey \& Hall, 1989; Miller, 1971) indican que, al examinar estímulos formados por elementos de modalidades sensoriales distintas, la respuesta a dicho estímulo será más parecida a un agregado de las representaciones de aquellos elementos que lo componen que cuando este está compuesto por elementos de la misma modalidad sensorial. Se ha sugerido que esto podría deberse a cierto tipo de interacción perceptiva, propia de los compuestos intradimesionales, en la que la presentación conjunta de estímulos alteraría sus propiedades perceptivas, lo cual es congruente con otros datos, tales como aquellos reportados por Bahçekapili (1997) en el aprendizaje de ciertas discriminaciones con estímulos auditivos que poseen distintos grados de interacción perceptual.

Una de las formas en la que podría ocurrir la interacción perceptiva se relaciona con la distinción entre dimensiones integrales y separables propuesta por Garner (1970). Según este autor, dos dimensiones son integrales si para que una de las dimensiones exista debe existir necesariamente algún nivel de la otra (e.g., saturación y brillo), mientras que dos dimensiones son separables si es posible percibir o atender a una sola dimensión sin atender a la otra (e.g., tamaño de un círculo y ángulo de su radio). Una consecuencia práctica de esta distinción es que las dimensiones integrales se interfieren entre sí mucho más que las dimensiones separables, de tal manera que en un estímulo formado por dos dimensiones integrales la identidad de cada dimensión tiende a perderse, generándose una nueva fusión, mientras que en las separables hay una conservación de la identidad de cada dimensión. Siguiendo esta línea de razonamiento, varios autores han propuesto que 
la distinción entre dimensiones integrales y separables podría ser una de las fuentes de la llamada flexibilidad de procesamiento en el aprendizaje asociativo (Bahçekapili, 1997; Lachnit, 1988; Melchers et al., 2008; Myers, Vogel, Shin \& Wagner, 2001). Sin embargo, muy pocos estudios han examinado sistemáticamente esta posibilidad.

En un intento por conectar las nociones de integralidad y separabilidad con el debate acerca de la flexibilidad de procesamiento en el condicionamiento, Lachnit (1988) investigó un procedimiento de discriminación en el condicionamiento galvánico de la piel en humanos, manipulando las características de los estímulos. Sus resultados indicaron un procesamiento más bien configuracional, es decir, alta sensibilidad contextual cuando las dimensiones utilizadas eran del tipo integral (saturación y brillo de una imagen). Por el contrario, sus hallazgos fueron del tipo elementalista, es decir, baja sensibilidad contextual, cuando las dimensiones utilizadas eran del tipo separable (tamaño de un circulo y ángulo de su radio).

Llama la atención que muy pocos estudios hayan continuado explorando la posible influencia de la integralidad versus separabilidad de la dimensiones en el procesamiento de los estímulos (e.g., Thorwart \& Lachnit, 2009). La validez externa de estos hallazgos requiere que se examinen estos efectos con otros sistemas de respuesta, aparte de la respuesta galvánica, y con otros procesos de aprendizaje. Con el fin de responder a esta necesidad, la presente investigación pretendió examinar la generalidad de los hallazgos de Lachnit (1988), utilizando el paradigma de aprendizaje predictivo humano, el cual ha sido frecuentemente utilizado como herramienta de evaluación de modelos de aprendizaje asociativo en humanos. Específicamente, el objetivo de la presente investigación fue determinar si el tipo de dimensiones utilizadas para construir los estímulos (integral versus separable) determina el tipo de procesamiento (configuracional o elementalista) que ocurre en el aprendizaje de relaciones predictivas en humanos.

\section{Método}

\section{Participantes}

Un total de 38 estudiantes de psicología de la Universidad de Talca, sin experiencia previa en una investigación similar, participó en el experimento, como parte de un requisito de un módulo del curso Metodología de la Investigación. La edad media fue 18,2 años $(D E=0,2)$. Los participantes fueron examinados individualmente y asignados aleatoriamente a uno de los dos grupos (integral y separable, $n=19$ ).

\section{Procedimiento y Materiales}

Los participantes firmaron una carta de consentimiento informado, aprobada por el comité de ética de la Universidad de Talca y por el Fondo Nacional de Desarrollo Científico y Tecnológico.

Para la realización del experimento cada participante fue dejado solo en una habitación con un computador, en el cual se le presentaron todas las instrucciones necesarias. Para esto se utilizó un computador HP Compaq con una pantalla de 14 pulgadas, siendo programado con el programa E-Prime (Versión 1.1). Los participantes emitieron sus respuestas seleccionando íconos con el ratón del computador.

La situación experimental consiste en que cada participante debe suponer que es un alergista quien tiene que aprender qué microorganismos producen una reacción alérgica al aplicar en la piel de animales de laboratorio ficticios un producto orgánico cuyo componente principal son distintos microorganismos. Para esto se construyeron cuatro dibujos de microorganismos (claves experimentales) a partir de la combinación de distintos niveles de dos dimensiones (brillo y saturación para el grupo integral y tamaño del círculo y ángulo de su línea radial para el grupo separable). En la fase de entrenamiento los estímulos a1b2 y a3b4 siempre fueron seguidos por la consecuencia (reforzados), mientras que los estímulos a2b1y a4b3 nunca fueron seguidos por la consecuencia (no reforzados). La fase de prueba contempló que los participantes estimaran 
el valor predictivo de las claves novedosas a2b3 y a3b2, así como también de las claves entrenadas. De replicarse los hallazgos de Lachnit (1988), se esperaba que en el grupo separable las respuestas a los compuestos novedosos se parecieran mucho más que en el grupo integral a las de las claves que contienen sus elementos, denotando el último grupo una mayor sensibilidad contextual.

Los estímulos utilizados en la condición separable fueron círculos blancos de diferentes diámetros $(\mathrm{a} 1=0,9 \mathrm{~cm}, \mathrm{a} 2=1,2 \mathrm{~cm}, \mathrm{a} 3$ $=2,9 \mathrm{~cm}$ y a $4=5,2 \mathrm{~cm}$ ) con líneas radiales de diferentes ángulos $\left(\mathrm{b} 1=0^{\circ}, \mathrm{b} 2=90^{\circ}, \mathrm{b} 3\right.$ $=180^{\circ}$ y b4 $=270^{\circ}$ ). Los estímulos de la condición integral fueron colores de un matiz 5 rojo púrpura bajo las tinturas de Munsell con diferentes valores de brillo $(\mathrm{a} 1=4 /, \mathrm{a} 2=$ $5 /$, a $3=6 /$ y a $4=7 /)$ y saturación $(\mathrm{b} 1=/ 6, \mathrm{~b} 2$ $=/ 8, \mathrm{~b} 3=/ 10 \mathrm{y} \mathrm{b} 4=/ 12)$.

Los estímulos separables e integrales fueron creados usando los programas Cabri 3D (Versión 2) y FreeHand MX (Versión 11), respectivamente. Todos los estímulos fueron presentados dentro de una figura en forma de célula de contorno irregular de aproximadamente $6,4 \times 7,8 \mathrm{~cm}$.

Durante la fase de entrenamiento, una serie de 80 ensayos fue presentada a los participantes. Al comienzo de cada ensayo, la oración "La muestra contiene el siguiente microorganismo" apareció en la esquina superior izquierda de la pantalla, seguida por los estímulos. La presentación del estímulo fue seguida por la pregunta "¿Piensas que la rata desarrollaría reacción alérgica?", la cual era seguida por la oración "Presiona SÍ para indicar una reacción alérgica y NO para indicar sin reacción alérgica”. Después de que el participante daba su respuesta, se entregaba retroalimentación en la parte inferior de la pantalla por 4,5 segundos. Esta consistía en las palabras "CORRECTO"/ "INCORRECTO" en amarillo, sobre la sentencia en rojo "Alergia" para los ensayos de $\mathrm{a} 1 \mathrm{~b} 2+$ y a3b4+ o en verde "No Alergia" para los ensayos de a2b1- y a4b3-. Adicionalmente, se daba una retroalimentación cuantitativa con la oración "0 puntos de alergia sobre un total de 100" o "50 puntos de alergia sobre un total de 100", debajo de las frases "No Alergia" y "Alergia", respectivamente. Los paneles superior e intermedio de la Fi- gura 1 presentan un ejemplo de un ensayo de entrenamiento y su correspondiente retroalimentación. El ensayo termina con una nueva pantalla de un segundo de duración reportando el porcentaje acumulado de respuestas correctas. Cada tipo de ensayo se presentó en orden aleatorio una vez dentro de cada bloque de cuatro ensayos, totalizando 20 ensayos de cada tipo por cada clave.

$\mathrm{Al}$ completar los 80 ensayos de entrenamiento, se les presentó a los participantes el siguiente mensaje: "A continuación, queremos que estimes la probabilidad de que cada muestra de microorganismos cause una reacción alérgica en las ratas". Durante esta fase, cada estímulo apareció separadamente en el centro superior de la pantalla y se le pidió al participante que "estime el grado de reacción alérgica que este microorganismo producirá". Para dar su respuesta, el participante debía escoger un número entre 0 y 100 de una escala de 11 puntos. A los participantes se les pidió evaluar las cuatro claves entrenadas (a1b2, a2b1, a3b4 y a4b3), junto a dos compuestos novedosos (a3b2 y a2b3), los cuales se presentaron en orden aleatorio. No se entregó ningún tipo de retroalimentación durante esa fase. El panel inferior de la Figura 1 muestra un ejemplo de uno de estos ensayos de prueba.

\section{Análisis Estadístico}

La variable dependiente del estudio fue la puntuación (juicio) predictiva asignada por los participantes a cada una de las claves en la fase de prueba. Los juicios causales fueron promediados y sometidos a un ANOVA mixto de 2 (Grupo: integral y separable) X 6 (Clave: a1b2, a3b4, a2b1, a4b3, a2b3 y a3b2). El factor Grupo es un factor intersujetos y el factor Clave es intrasujetos. Las posibles diferencias entre pares de claves fueron examinadas a través de comparaciones post-hoc con la corrección de Bonferroni.

Se examinó el supuesto de igualdad de varianzas por medio de la prueba de esfericidad de Mauchly, encontrándose falta de apoyo para dicho supuesto, $W(14)=0,34, p=$ 0,001 . Consecuentemente, se corrigieron los grados de libertad de la prueba $F$, de acuerdo a la técnica de Greenhouse-Geisser. 


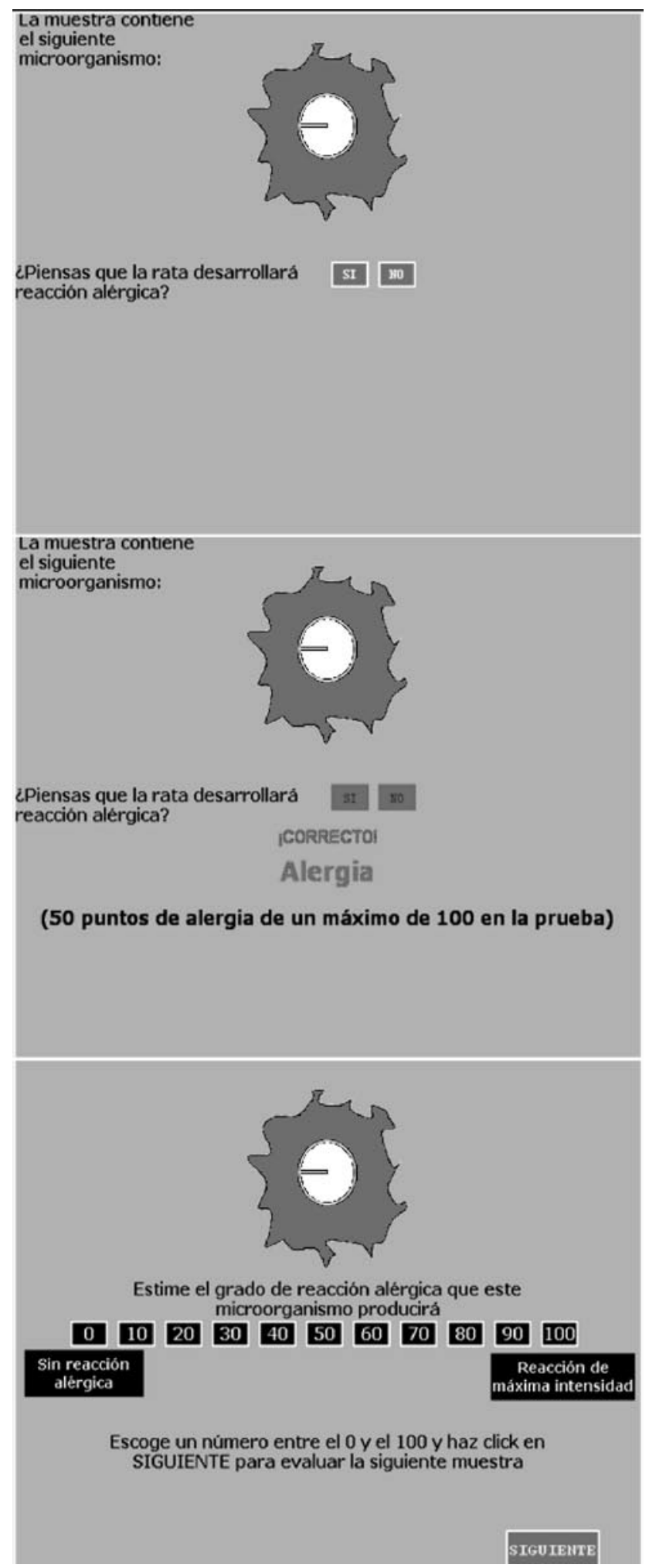

Figura 1. Ejemplo de las pantallas presentadas a los participantes durante el entrenamiento (panel superior e intermedio) y la prueba (panel inferior). 


\section{Resultados}

La Figura 2 presenta el promedio de los juicios predictivos para las claves reforzadas (a1b2 y a3b4), no reforzadas (a2b1 y a4b3) y novedosas (a2b3 y a3b2) en ambos grupos. La figura indica que ambos grupos aprendieron la discriminación al otorgar mayores puntajes predictivos promedio a las claves reforzadas que a las no reforzadas. Adicionalmente, los datos sugieren que el puntaje promedio asignado por el grupo integral a la clave formada por elementos reforzados (a3b2) fue muy inferior que aquel asignado a las claves reforzadas (a1b2 y a3b4), lo que no se produjo de manera tan marcada en el grupo separable. Esto apoya la hipótesis de una mayor sensibilidad contextual en la condición integral que en la separable. Análogamente, las puntuaciones para las claves novedosas formadas por elementos no reforzados están en concordancia con esta conclusión, ya que los puntajes del grupo integral para la clave a2b3 fueron muy superiores a los de las claves no reforzadas, lo que no se observa en el grupo separable. En resumen, los puntajes para las claves nove- dosas fueron muy similares a los de las claves entrenadas respectivas en la condición separable, pero no en la integral.

El análisis de varianza confirma estas observaciones, ya que arrojó un efecto principal significativo de Clave, $F(5,180)=$ 83,38, $p<0,001, \eta^{2}=0,70,95 \%$ ICs [49,0, $63,8],[56,4,70,2],[2,2,11,8],[1,1,11,6]$, $[19,7,34,2]$ y $[27,2,37,5]$, para las claves a1b2, a3b4, a2b1, a4b3, a2b3 y a3b2, respectivamente, y una interacción significativa entre Clave y Grupo, $F(5,180)=39,07, p<$ $0,001, \eta_{\mathrm{p}}^{2}=0,52,95 \% \operatorname{ICs}[44,3,65,2],[54,4$, $74,0],[2,5,16,0],[2,3,17,2],[40,3,60,8] \mathrm{y}$ [-2.8, 11,7], para las claves a1b2, a3b4, a2b1, a4b3, a2b3 y a3b2, respectivamente del grupo integral, y $[47,7,68,6],[52,6$, $72.2],[-2,0,11,5],[-4.5,10,33],[-6,8,13,7]$ y $[53,0,67.5]$, para las claves a1b2, a3b4, a2b1, a4b3, a2b3 y a3b2, respectivamente del grupo separable. Tanto el efecto de Clave como la interacción entre Clave y Grupo alcanzaron potencias observadas mayores a 0,99 , indicando la alta replicabilidad de estos efectos.

El efecto principal de Clave se debe a las bajas puntuaciones tanto de las claves

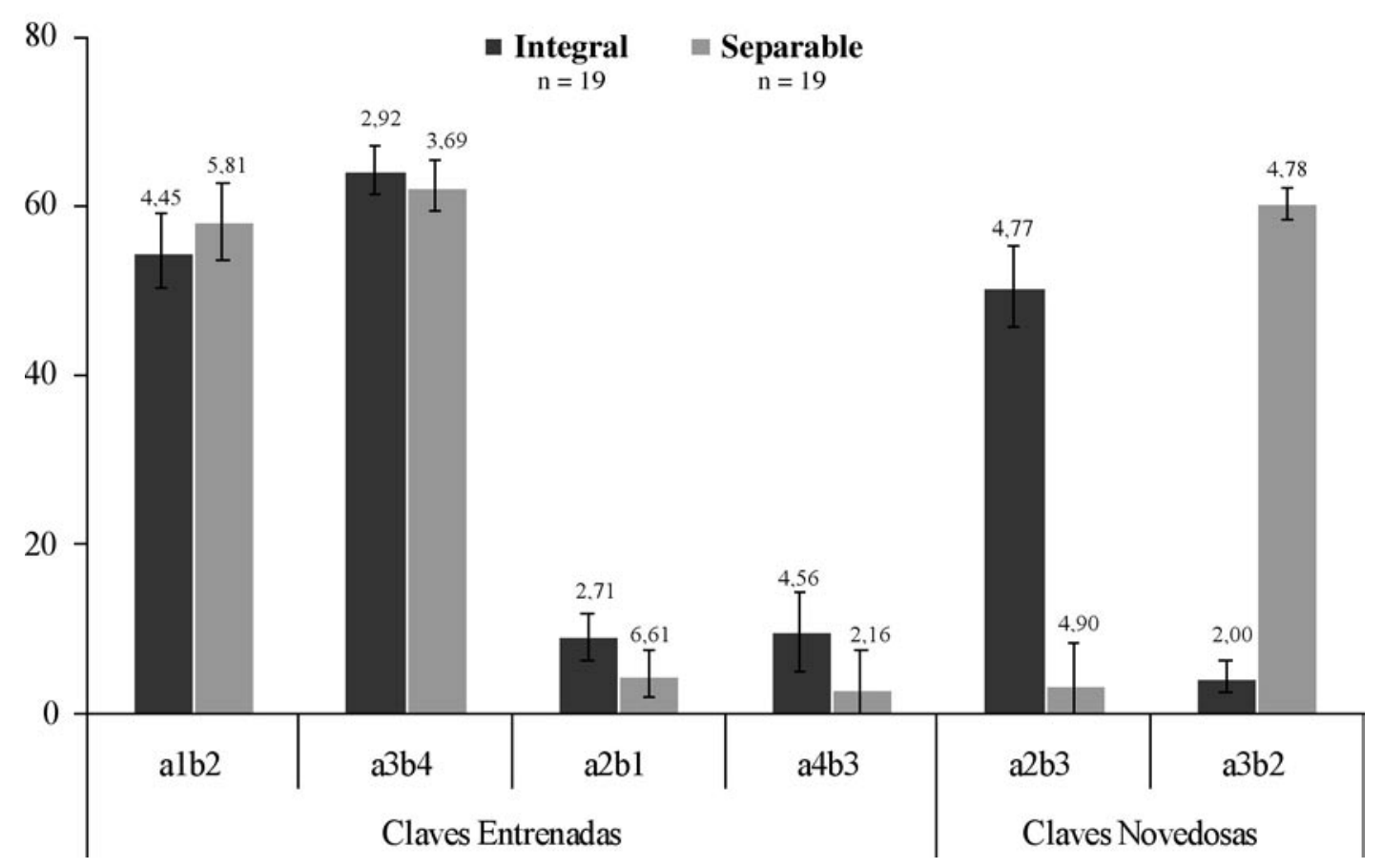

Figura 2. Media de juicios predictivos asignados en el experimento a cada clave durante la prueba. Las barras de error representan el error estándar de la media. 
entrenadas como no reforzadas, en comparación a las de las claves entrenadas y reforzadas en ambos grupos. Esto fue confirmado por las comparaciones por pares, las cuales indicaron que las puntuaciones medias otorgadas a las claves a $1 b 2$ y a $3 b 4$ no difirieron significativamente entre sí ( $p=$ $0,171)$, pero fueron significativamente mayores que las puntuaciones promedio asignadas a a2b1 y a4b3 $(p<0,001)$, las cuales a su vez no difirieron significativamente entre sí $(p>0,999)$.

La interacción entre Clave y Grupo fue examinada a través de comparaciones por pares entre ambos grupos respecto a sus puntuaciones para cada clave. Este análisis indicó que los grupos no difirieron significativamente en sus puntuaciones a las claves entrenadas $(p>0,195)$, pero sí se diferenciaron significativamente en las puntuaciones a las claves novedosas. Específicamente, las puntuaciones a la clave novedosa a3b2 fueron mayores en el grupo separable que en el integral $(p<0,001)$ y las puntuaciones a la clave novedosa a2b3 fueron mayores en el grupo integral que en el grupo separable ( $p$ $<0,001$ ), indicando que los datos del grupo integral fueron más sensibles al contexto que los del grupo separable.

Además del análisis anterior, la evidencia más directa de diferencias en la sensibilidad contextual de ambos grupos se puede apreciar en el hecho de que la clave a3b2 no difirió significativamente de las claves a1b2 $\mathrm{y}$ a3b4 en el grupo separable $(p>0,999)$, pero fue significativamente inferior a estas en el grupo integral $(p<0,001)$. Una situación análoga ocurrió con la clave novedosa compuesta por elementos negativos (a2b3), ya que esta no difirió significativamente de las claves entrenadas como negativas en el grupo separable $(p>0,999)$, pero difirió significativamente de estas en el grupo integral $(p<0,001)$.

En resumen, estos resultados indican que cuando se utilizan dimensiones que se relacionan entre sí de una manera integral, al formar un compuesto novedoso (a3b2) a partir de elementos que han sido previamente entrenados separadamente como predictores de una consecuencia (a1b2+y a $3 \mathrm{~b} 4+$ ) o formando un compuesto novedoso con elementos que han sido previamente entrenados separadamente como no predictores de una consecuencia (a2b1- y a4b3-), resulta en un juicio predictivo a dichas claves que no puede ser completamente explicado en función del valor predictivo de sus elementos. Por el contrario, cuando se utilizan dimensiones separables, los valores de las claves novedosas pueden ser explicados íntegramente en términos de las asociaciones adquiridas por sus elementos.

\section{Discusión}

El presente estudio demostró que en el aprendizaje predictivo existe una mayor sensibilidad contextual cuando los estímulos están compuestos por dimensiones integrales que cuando están compuestos por dimensiones separables. De este modo, los resultados de este estudio proporcionan evidencia consistente con la hipótesis de que las propiedades psicofísicas de los estímulos juegan un rol fundamental en el tipo de procesamiento que se hace en situaciones de aprendizaje asociativo. Otra forma de referirse a estos resultados es señalando que, al juntar dos estímulos de tipo integral, el resultado es un todo único indivisible o configuración, mientras que, al juntar dos estímulos de tipo separable, el resultado es una agregación de elementos.

Estos hallazgos son virtualmente idénticos a los resultados obtenidos por Lachnit (1988, Experimento 2), quien utilizó el mismo diseño experimental y estímulos que en la presente investigación, pero aplicados a una tarea de condicionamiento pavloviano de la respuesta galvánica en humanos. Específicamente, en el experimento de Lachnit (1988) las claves a $1 b 2$ y a3b4 fueron reforzadas con una leve estimulación eléctrica en la muñeca (estímulo incondicionado), mientras que las claves a $2 \mathrm{~b} 1$ y a $4 \mathrm{~b} 3$ no fueron reforzadas. Los resultados de la fase de prueba con los estímulos novedosos indicaron más respuestas condicionadas a a $3 b 2$ que a a $2 b 3$ en el grupo entrenado con dimensiones separables y más respuestas condicionadas a a2b3 que a a3b2 en el grupo entrenado con dimensiones integrales. Estos hallazgos fueron interpretados por Lachnit (1988) señalando que las respuestas de los participantes entrenados con dimensiones separables 
estuvieron más influidas por los elementos constituyentes de cada compuesto que las respuestas de los participantes entrenados con estímulos pertenecientes a dimensiones integrales, las cuales parecieron estar más bien determinadas por la mayor similitud global de a3b2 con las claves no reforzadas y de a2b3 con las claves reforzadas.

Pese a lo promisorio de los hallazgos de Lachnit (1988), es importante consignar que algunas comparaciones relevantes no fueron reportadas en su estudio. La principal omisión se refiere a que la sensibilidad contextual diferencial en los grupos integral y separable se infirió indirectamente a través de una comparación entre la clave novedosa formada por elementos positivos (a3b2) y la clave novedosa formada por elementos negativos (a2b3). La comparación más directa de este efecto debería haber sido entre cada clave entrenada y las correspondientes claves que contenían sus elementos. Así, por ejemplo, una mayor sensibilidad contextual en el grupo integral que en el separable debería implicar una mayor diferencia entre los compuestos positivos (a $1 b 2$ y a3b4) y la clave a3b2. Esta omisión fue subsanada en la presente investigación, puesto que en la fase de prueba se examinaron las asociaciones de las cuatro claves entrenadas y las dos claves novedosas.

Tomados en conjunto, los hallazgos de Lachnit (1988) y los obtenidos en la presente investigación contribuyen a la generalidad de la hipótesis de la flexibilidad de procesamiento. En este contexto, es interesante considerar el isomorfismo que existe entre el condicionamiento y el aprendizaje predictivo, ya que los eventos predictivos o claves cumplen el mismo rol que los estímulos condicionados, la consecuencia a predecir es análoga al estímulo incondicionado y el juicio predictivo hace las veces de la respuesta condicionada. Al existir congruencia de resultados en los experimentos realizados con ambos procedimientos, adquiere fuerza la idea de que ambas formas de aprendizaje podrían estar reguladas por mecanismos asociativos similares.

Desde una perspectiva global, estos hallazgos de variabilidad de procesamiento en el condicionamiento y el aprendizaje predictivo imponen un interesante desafío para los modelos de aprendizaje asociativo. Como se mencionó en la introducción, la mayoría de estos modelos implementa la sensibilidad contextual, creando estructuras representacionales que posibilitan que los estímulos experimentalmente definidos por los investigadores cambien en función de la presencia o ausencia de otros estímulos (Harris, 2006; Wagner, 2003). Sin embargo, con el fin de dar cuenta de las diferencias en el grado de especificidad contextual entre estímulos de distinto tipo, los modelos deben suponer además que el grado de sensibilidad contextual varía dependiendo de la naturaleza de los estímulos. Una posibilidad es el modelo planteado por Wagner y sus colaboradores (Brandon, Vogel \& Wagner, 2000; Vogel et al., 2007; Wagner, 2003). En este modelo se adopta una postura componencial (del inglés componential), en la cual los estímulos están formados por un conjunto de elementos, cuya actividad, aparte de depender del estímulo que representan, depende en grados variables de la presencia de otros estímulos. Según esta lógica, el grado de sensibilidad contextual del modelo puede emerger naturalmente de la proporción relativa de elementos dependientes e independientes del contexto. Asimismo, las diferencias obtenidas en la presente investigación entre dimensiones separables e integrales podrían explicarse por diferencias en las proporciones relativas de estos tipos de elementos en las dos condiciones experimentales.

A manera de ejemplo, en la Figura 3 se presentan las simulaciones computacionales para los resultados de la presente investigación con el modelo de elementos reemplazados (REM), según los algoritmos y el programa computacional propuestos por Vogel et al. (2007). En estas simulaciones la sensibilidad contextual depende de un parámetro, $\mathrm{r}$, el cual varía entre 0 (muy baja sensibilidad contextual) y 1 ( $m$ uy alta sensibilidad contextual). Allí se aprecia que cuando la proporción de elementos dependientes del contexto es moderadamente alta $(r=0,7)$ se predice una gran disminución de la fuerza asociativa del compuesto novedoso, en comparación con las simulaciones que utilizan un valor de reemplazo nulo $(r=0)$, en las cuales no hay disminución alguna. 


$$
\text { - Integral }(\mathrm{r}=0,7) \square \text { Separable }(\mathrm{r}=0)
$$

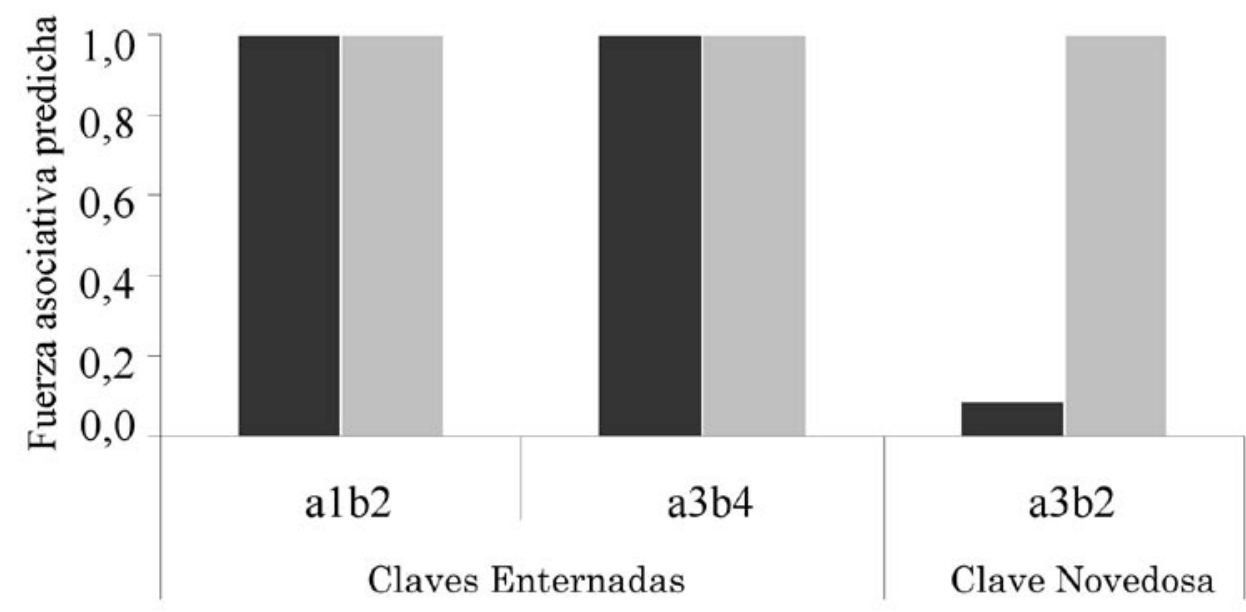

Figura 3. Simulaciones computacionales realizadas con el modelo REM (Wagner, 2003) para las claves reforzadas y la clave novedosa a3b2, tanto para la condición integral como separable. El nivel de sensibilidad contextual fue $\mathrm{r}=0$ y $\mathrm{r}=0,7$ para los estímulos separables e integrales, respectivamente.

Este mismo principio de reemplazo diferencial para estímulos de distinta naturaleza podría aplicarse para dar cuenta de los datos reportados por Kehoe et al. (1994), en los que se observa sumatoria con estímulos pertenecientes a distintas modalidades sensoriales (estableciendo una baja proporción de elementos dependientes del contexto) y ausencia de sumatoria con estímulos de la misma modalidad sensorial (estableciendo una alta proporción de elementos dependientes del contexto).

Es importante consignar que, si bien los parámetros utilizados en las simulaciones de la Figura 3 describen apropiadamente los datos de la presente investigación, no pueden dar cuenta de todos los hallazgos observados en la literatura. Por ejemplo, otras investigaciones en el ámbito del aprendizaje predictivo (Cornejo et al., 2010; Díaz et al., 2009) han concluido que, al utilizar estímulos con formas complejas y significativas (figuras humanoides y alimentos), el parámetro que mejor da cuenta de sus resultados es un valor intermedio de sensibilidad contextual $(r=0,3)$. Claramente se requiere de más investigación y de una mayor elaboración teórica acerca del significado de la sensibilidad contextual para comprender la naturaleza de estas diferencias.
Algunos modelos recientes han aventurado algunas hipótesis acerca de cómo podría operar esta mayor o menor sensibilidad contextual que Wagner articula a través de su parámetro r. Por ejemplo, Harris y sus colaboradores (Harris, 2006, 2010; Harris \& Livesey, 2010) plantean que la sensibilidad contextual emerge de la competencia por niveles de activación o por la atención de la que gozan los distintos elementos que representan a los estímulos y que esta competencia afecta más a algunos elementos que a otros. Específicamente, Harris y sus colaboradores sugieren que cada estímulo está representado por un conjunto único de elementos cuyos niveles de activación varían siguiendo una función sigmoidea. Se asume, además, que el sistema tiene una capacidad limitada, de tal manera que los elementos sufrirán un proceso de normalización, disminuyendo sus niveles de activación en función de la cantidad neta de estimulación. Esta normalización afecta menos a aquellos elementos que se encuentran en los extremos de la función sigmoidea que a los del centro, siendo estos últimos aquellos elementos de los cuales emerge la sensibilidad contextual. Al igual que en el modelo REM, uno podría asumir que la cantidad de normalización varía dependiendo de la 
naturaleza de los estímulos, lo cual puede también explicar los resultados de la presente investigación.

Pese a que Harris (2006), Wagner (2003) y otros autores que han hecho propuestas similares (e.g., McLaren \& Mackintosh, 2002) pueden describir bastante bien los datos, apelando a cambios paramétricos, es importante consignar que estos modelos no predicen los datos. Predicciones precisas en este ámbito surgirán en la medida que se mejore la comprensión teórica y empírica de estos fenómenos.

Quedan pendientes interrogantes, tales como: ¿por qué se produce esta variación en la sensibilidad contextual en situaciones experimentales como las de la investigación reportada en este artículo? y ¿qué tienen en común estos hallazgos con la supuesta variabilidad de procesamiento observada por Kehoe et al. (1994) con compuestos intradimensionales y extradimensionales? Asimismo, futuros estudios deben investigar otras variables que podrían influir en el tipo de procesamiento de los estímulos en un variado rango de tareas experimentales (Cornejo et al., 2010; Díaz et al., 2009; Williams, Sagness \& McPhee, 1994).

\section{Referencias}

Aydin, A. \& Pearce, J. M. (1995). Summation in autoshaping with short- and long-duration stimuli. Quarterly Journal of Experimental Psychology: Section B, 48, 215-234. doi:10.1080/1 4640749508401449

Bahçekapili, H. G. (1997). An evaluation of Rescorla and Wagner's elementistic model versus Pearce's configural model in discrimination learning (Tesis de Doctorado no publicada), Yale University, New Heaven, CT.

Brandon, S. E., Vogel, E. H. \& Wagner, A. R. (2000). A componential view of configural cues in generalization and discrimination in Pavlovian conditioning. Behavioural Brain Research, 110, 67-72. doi:10.1016/S0166-4328(99)00185-0

Cabri 3D (Versión 2) [Software computacional]. Grenoble, Francia: Cabrilog.

Collins, D. J. \& Shanks, D. R. (2006). Summation in causal learning: Elemental processing or configural generalization? Quarterly Journal of Experimental Psychology, 59, 1524-1534. doi:10. 1080/17470210600639389

Cornejo, F. A., Castillo, R. D., Saavedra, M. A. \& Vogel, E. H. (2010). Summation in predictive learning in children. Psicológica, 31, 199-217.
Díaz, A. C., Torres, L. D., Castillo, R. D., Cornejo, F. A. \& Vogel, E. H. (2009). Estrategias de codificación de estímulos en el aprendizaje causal humano. Psykhe, 18(2), 79-96. doi:10.4067/S071822282009000200006

E-Prime (Versión 1.1) [Software computacional]. Pittsburgh, PA: Psychology Software Tools.

FreeHand MX (Versión 11) [Software computacional]. San Jose, CA: Adobe Systems.

Garner, W. R. (1970). The stimulus in information processing. American Psychologist, 25, 350-358. doi:10.1037/h0029456

Harris, J. A. (2006). Elemental representations of stimuli in associative learning. Psychological Review, 113, 584-605. doi:10.1037/0033-295X.113.3.584

Harris, J. A. (2010). The arguments of associations. En N. A. Schmajuk (Ed.), Computational models of conditioning (pp. 53-70). Cambridge, Reino Unido: Cambridge University Press.

Harris, J. A. \& Livesey, E. J. (2008). Comparing patterning and biconditional discriminations in humans. Journal of Experimental Psychology: Animal Behavior Processes, 34, 144-154. doi:10.1037/0097-7403.34.1.144

Harris, J. A. \& Livesey, E. J. (2010). An attention modulated associative network. Learning \& Behavior, 38, 1-26. doi:10.3758/LB.38.1.1

Harris, J. A., Livesey, E. J., Gharaei, S. \& Westbrook, R. F. (2008). Negative patterning is easier than a biconditional discrimination. Journal of Experimental Psychology: Animal Behavior Processes, 34, 494-500. doi:10.1037/0097-7403.34.4.494

Honey, R. C. \& Hall, G. (1989). Attenuation of latent inhibition after compound pre-exposure: Associative and perceptual explanations. Quarterly Journal of Experimental Psychology: Section B, 41, 355-368. doi:10.1080/1464074890 8401201

Kehoe, E. J., Horne, A. J., Horne, P. S. \& Macrae, M. (1994). Summation and configuration between and within sensory modalities in classical conditioning of the rabbit. Animal Learning \& Behavior, 22, 19-26.

Lachnit, H. (1988). Convergent validation of information processing constructs with Pavlovian methodology. Journal of Experimental Psychology: Human Perception and Performance, 14, 143-152. doi:10.1037/0096-1523.14.1.143

Ludwig, I. \& Lachnit H. (2003). Asymmetric interference in patterning discriminations: A case of modulated attention. Biological Psychology, 62, 133-146. doi:10.1016/S0301-0511(02)00124-2

McLaren, I. P. L. \& Mackintosh, N. J. (2002). Associative learning and elemental representation: II. Generalization and discrimination. Animal Learning \& Behavior, 30, 177-200.

Melchers, K. G., Shanks, D. R. \& Lachnit, H. (2008). Stimulus coding in human associative learning: Flexible representations of parts and wholes. Behavioural Processes, 77, 413-427. doi:10.1016/ j.beproc.2007.09.013

Miller, L. (1971). Compounding of discriminative stimuli from the same and different sensory modalities. Journal of Experimental Psychology: Animal Behavior Processes, 16, 337-342. 
Myers, K. M., Vogel, E. H., Shin, J. \& Wagner, A. R. (2001). A comparison of the Rescorla-Wagner and Pearce models in a negative patterning and a summation problem. Animal Learning \& Behavior, 29, 36-45.

Pearce, J. M. (2002). Evaluation and development of a connectionist theory of configural learning. Animal Learning \& Behavior, 30, 73-95.

Pearce, J. M. \& Wilson, P. N. (1991). Failure of excitatory conditioning to extinguish the influence of a conditioned inhibitor. Journal of Experimental Psychology: Animal Behavior Processes, 17, 519529. doi:10.1037/0097-7403.17.4.519

Rescorla, R. A. (1997). Summation: Assessment of a configural theory. Animal Learning \& Behavior, 25, 200-209.

Rescorla, R. A. \& Coldwell, S. E. (1995). Summation in autoshaping. Animal Learning \& Behavior, 23, 314-326.

Saavedra, M. A. (1975). Pavlovian compound conditioning in the rabbit. Learning and Motivation, 6, 314-326. doi:10.1016/0023-9690(75)90012-0

Soto, F. A., Vogel, E. H., Castillo, R. D. \& Wagner, A. R. (2009). Generality of the summation effect in human causal learning. Quarterly Journal of Experimental Psychology, 62, 877-889. doi:10.108 0/17470210802373688

Thorwart, A. \& Lachnit, H. (2009). Symmetrical generalization decrements: Configural stimulus processing in human contingency learning. Learning \& Behavior, 37, 107-115. doi:10.3758/ LB.37.1.107

Vogel, E. H., Díaz, C. A., Ramírez, J. A., Jarur, M. C., Pérez-Acosta, A. M. \& Wagner, A. R. (2007).
Desarrollo de un programa computacional para simular las predicciones del modelo de elementos reemplazados (REM) de condicionamiento pavloviano. Psicothema, 19, 506-514.

Vogel, E. H. \& Ponce, F. P. (en prensa). Mechanisms of Pavlovian conditioning. En E. Alonso \& E. Mondragón (Eds.), Computational neuroscience for advancing artificial intelligence: Models, methods and applications. Hershey, PA: IGI Global.

Wagner, A. R. (2003). Context-sensitive elemental theory. Quarterly Journal of Experimental Psychology: Section B, 56, 7-29. doi:10.1080/027 24990244000133

Wagner, A. R. \& Rescorla, R. A. (1972). Inhibition in Pavlovian conditioning: Application of a theory. En M. S. Halliday \& R. A. Boakes (Eds.), Inhibition and learning (pp. 301-336). San Diego, CA: Academic Press.

Wagner, A. R. \& Vogel, E. H., (2009). Conditioning theories. En L. R. Squire (Ed.), Encyclopedia of neuroscience (Vol. 3, pp 49-57). Oxford, Reino Unido: Academic Press. doi:10.1016/B978-0080450469.01555-2

Whitlow, J. W. \& Wagner, A. R. (1972). Negative patterning in classical conditioning: Summation of response tendencies to isolable and configural components. Psychonomic Science, 27, 299-301.

Williams, D. A., Sagness, K. E. \& McPhee, J. E. (1994). Configural and elemental strategies in predictive learning. Journal of Experimental Psychology: Learning, Memory and Cognition, 20, 694-709. doi:10.1037/0278-7393.20.3.694

Fecha de recepción: Mayo de 2010.

Fecha de aceptación: Octubre de 2010. 